\title{
Role for ezrin in breast cancer cell chemotaxis to CCL5
}

\author{
JUANJUAN LI*, YI TU*, JING WEN*, FENG YAO, WEN WEI and SHENGRONG SUN \\ Department of Breast and Thyroid Gland Surgery of Renmin Hospital of Wuhan University, \\ The First College of Clinical Medicine of Wuhan University, Wuhan, Hubei 430060, P.R. China
}

Received March 23, 2010; Accepted June 11, 2010

DOI: $10.3892 /$ or_00000943

\begin{abstract}
This study was undertaken to observe the effects and the possible mechanism of membrane-cytoskeleton linker ezrin on the chemokine CC chemokine ligand 5 (CCL5)induced invasive ability in human breast carcinoma MCF-7 cells. RNA interference (RNAi) using ezrin small hairpin RNAs (ezrin shRNA) was used to analyze the role of ezrin in the regulation of this CCL5-induced malignant behavior of MCF-7 cells. The effects of recombinant human CCL5 (rhCCL5) on the cell's invasive ability were detected by transwell assay. Western blotting was performed to examine the expression of the total and the phosphorylated ezrin at protein level. The CCL5-induced changes in the organization of the actin cytoskeleton in the transfected cells were determined using confocal microscopy. Flow cytometry was used to detect the cell cycle. MTT method was used to detect the proliferation of the cells. We found that the MCF-7 cells responded chemotactically to CCL5 in a dose- and timedependent manner. After RNAi treatment, the proliferation was inhibited and the cell proportion in G2-M phase decreased. The CCL5-induced cell motility and invasiveness were obviously inhibited by the silencing of ezrin. In addition, the CCL5 induced a significant up-regulation in the total and the phosphorylated ezrin expression in MCF-7 cells, whereas in the presence of ezrin silencing, the CCL5 induced only a slight increase in the total and the phosphorylated ezrin expression. CCL5 was shown to induce changes in the organization of the actin cytoskeleton and the level of F-actin in MCF-7 cells, and the silencing of ezrin could inhibit these changes. Collectively, our data further show that CCL5 induced invasiveness in MCF-7 cells. These data indicate a potential role for ezrin in the processes of the CCL5-induced breast cancer cell migration, invasiveness and metastasis. It is suggested that ezrin may act as downstream effector of CCL5 and a new anti-invasive therapeutic target for human breast cancer.
\end{abstract}

Correspondence to: Dr Shengrong Sun, Department of Breast and Thyroid Surgery of Renmin Hospital of Wuhan University, The First College of Clinical Medicine of Wuhan University, 238 Jiefang Road, Wuhan, Hubei 430060, P.R. China

E-mail: sun137@sina.com

*Contributed equally

Key words: breast cancer, CC chemokine ligand 5, ezrin, invasion

\section{Introduction}

Breast cancer is one of the most common cancers in women all over the world. The poor prognosis of patients with breast cancer is related to the ability of tumor cells to metastasize to the regional lymph nodes, bone marrow, lungs and liver. Metastasis, a result of several sequential steps, represents a highly organized, non-random and organ-selective process (1). Tumor cell migration and metastasis share many similarities with the specific homing of haematopoietic cells to the specific sites, which is critically regulated by various factors in the microenvironment, including chemokines and their receptors (2-4).

CCL5, originally identified as a product of the activated $\mathrm{T}$ cells, is capable of recruiting $\mathrm{T}$ cells to inflammatory sites (5). The expression of the chemokine CCL5 by breast cancer is thought to correlate with the progression of the tumor, but the precise role is complex (6-10). CCL5 is expressed at high levels in some tissues (lungs, liver and bone marrow) that frequently harbor breast cancer metastasis. Investigating the association of CCL5 expression with the clinical course of breast cancer, the study showed that CCL5 levels in the patients with progression, relapse and metastasis were markedly increased compared to the patients in remission. Studies showed that the CCL5 expression was greater than that found in normal epithelial cells, ductal epithelial cells and benign sections $(8,11,12)$. It is conceivable that endogenously synthesized CCL5 may induce breast cancer cell migration, mediated by the receptor CCR5 (13). Several reports have demonstrated that blockade of CCR5 not only inhibited the metastatic process in vivo, but also produced a direct effect on both tumor progression and angiogenesis, which further implicates that CCL5 is an important molecule in breast cancer metastasis (14).

Ezrin, the coded product of the vil2 gene, is a member of the ERM (ezrin-radixin-moesin) cytoskeleton-associated protein family (15). Ezrin is best known as a key component that is involved in cell adhesion to the extracellular matrix, cell interactions, signal transduction, interactions with the cell apoptotic machinery (16-18), and cytoskeleton-mediated cell motility via controlling cell morphology $(19,20)$. Increasing mass of evidence indicates that ezrin is regarded as a metastatic determinant and a key component in tumor metastasis $(21,22)$. Yu et al reported that ezrin was consistently overexpressed in the metastatic clones from rhabdomyosarcoma and osteosarcomas $(23,24)$. Meanwhile, ezrin showed upregulated expression in a variety of invasive adult cancers, including uveal malignant melanomas (25), lungs (26) and 
endometrial carcinomas (27). Elliott et al found that ezrin was required for breast carcinoma metastasis (27). Transfection of ezrin constructs into low-metastatic cell lines dramatically increased the ability of these cell lines to form macroscopic pulmonary metastasis lesions, besides shRNA by RNA interference (RNAi) significantly reversed the metastatic behavior (29), suggesting that ezrin may be a key regulatory molecule in metastatic progression.

In this report, we showed that CCL5, an important chemotactic factor in breast cancer microenvironment, increased the invasive ability in MCF-7 cells in a dose-dependent manner. After the repression of ezrin in the stably expressing ezrin shRNAs MCF-7 cells, $>85 \%$ of $B$-actin was maintained at mRNA and protein level. After RNAi treatment, the proliferation was inhibited, and the cell proportion in G2-M phase decreased. We also discovered the CCL5-induced cell motility and invasiveness were obviously inhibited by the silencing of ezrin. At the same time, CCL5 markedly enhanced the expression of the total and the phosphorylated ezrin in MCF-7 cells, whereas in the presence of silenced ezrin, this increase was only slight. Besides, CCL5 was shown to induce changes in the organization of the actin cytoskeleton and the level of F-actin in MCF-7 cells, and the silencing of ezrin could inhibit these changes. Therefore, it is further revealed that CCL5 plays a significant role in the metastatic progress of breast cancer. Our results also show a potential role for ezrin in the CCL5-induced processes of the breast cancer cell migration, invasion and metastasis.

\section{Materials and methods}

Cell culture. MCF-7 cells were obtained from Type Culture Collection Center of Wuhan University, China. The cells were grown in RPMI-1640 supplemented with $10 \%$ heat-inactivated fetal calf serum (FCS, Gibco) and antibiotics (50 U/ml penicillin and $50 \mu \mathrm{g} / \mathrm{ml}$ streptomycin, Gibco) at $37^{\circ} \mathrm{C}$ in a humidified incubator with $5 \% \mathrm{CO}_{2}$.

$R T-P C R$. Total RNA was extracted from cell lines in TRIzol reagent (Invitrogen) and cDNA was synthesized from $1 \mu \mathrm{g}$ of total RNA using Superscript reverse transcriptase per the manufacturer's instructions. cDNA was subjected to PCR (GeneAmp PCR System 9700, Applied Biosystems) using ezrin specific, 5'-GAATACACAGCCAAGATTGC-3' (sense) and 5'-CTCATGTTCTCGTTGTGGAT-3' (antisense) primers, and GAPDH specific, 5'-GAATACACAGCCAAGATTGC-3' (sense) and 5'-CTCATGTTCTCGTTGTGGAT-3' (antisense) primers. PCR consisting of 30 cycles was carried out at the following conditions: at $95^{\circ} \mathrm{C}$ for $3 \mathrm{~min}, 45$ cycles at $94^{\circ} \mathrm{C}$ for $30 \mathrm{sec}$, annealing at $53^{\circ} \mathrm{C}$ for $30 \mathrm{sec}$ and elongation at $72^{\circ} \mathrm{C}$ for $30 \mathrm{sec}$. PCR products were analyzed on $1 \%$ agarose gel and visualized under UV light after staining with ethidium bromide.

Western blotting. Confluent cells were lysed in lysis buffer (20 mmol/1 Tris-HCl, pH 7.5, $150 \mathrm{mmol} / \mathrm{l}$ sodium chloride, $1 \mathrm{mmol} / \mathrm{l}$ EDTA, $1 \mathrm{mmol} / \mathrm{l}$ EGTA, $1 \%$ Triton, $2.5 \mathrm{mmol} / \mathrm{l}$ sodium pyrophosphate, $1 \mathrm{mmol} / \mathrm{l} \mathrm{h}$-glycerolphosphate, $1 \mathrm{mmol} / \mathrm{l}$ sodium orthovanadate, $0.5 \mathrm{mmol} / 1$ phenylmethylsulfonyl fluoride and $1 \mathrm{mg} / \mathrm{ml}$ leupeptin). Protein lysates (20-50 mg per lane), as determined by Bio-Rad protein assay, were separated in 10-12\% SDS-PAGE and then transferred to polyvinylidene difluoride membranes (Amersham Pharmacia Biotech). Membranes were blocked with $5 \%$ non-fat dried milk in TBS-T (20 mmol/1 Tris-HCl, pH 7.5, $8 \mathrm{~g} / \mathrm{l}$ of sodium chloride and $0.1 \%$ Tween-20) and then incubated with primary antibodies. Horseradish peroxidase conjugated anti-mouse IgG (Cell Signaling) was used as secondary antibody. Actin was used as internal positive control. Protein was visualized using enhanced chemiluminescence system (ECL, Amersham Pharmacia Biotech).

Plasmid construction and cell transfection. Small hairpin RNAs (shRNAs) of ezrin gene (no. of GenBank: NM_015873) were generated using an oligonucleotide DNA sequence. Two target sequences for effectively silencing ezrin expression were derived from the 553-571 nt and from $995 \mathrm{nt}$ to $1013 \mathrm{nt}$ positions of the coding region of the ezrin gene. Target sense sequence for Ezrin553, 5'-GATCCCCgataatgctatgttgga atTTCAAGAGAattccaacatagcattatcTTTTTa-3'; antisense, 5'-AGCTTAAAAAgataatgctatgttggaatTCTCTTGAAatt ccaacatagcattatc GGG-3'. Target sense sequence for Ezrin995, 5'-GATCCCCccgtggagagagagaaagaTTCAAGAGAtctttct ctctctccacggTTTTTa-3'; antisense, 5'-AGCTTAAAAAccgtg gagagagagaaagaTCTCTTGAAtctttctctctctccacggGGG-3' . The base pairs underlined in target sequences are the restriction sites HindIII and BamHI. The pSUPER plasmid was selected to express ezrin shRNAs.

The correctness of inserted sequences of the recombinants was confirmed by sequencing. According to the protocol supplied with the Lipofectamine transfection reagent (Life Technologies, Inc.), $2 \times 10^{5}$ cells were plated in 6-well dishes and incubated with the appropriate DNA and Lipofectamine 2000 in serum-free media for $5 \mathrm{~min}$, and then equal volumes of media containing $20 \%$ FCS were added. Stable shRNA clones were selected after 8-10 days of transfection for MTT cytotoxicity assay. RT-PCR and Western blotting were applied to analyze ezrin mRNA and protein levels, respectively.

Flow cytometric analysis. MCF-7 cells were seeded at a density of $2 \times 10^{5}$ per well in 6-well plates. After incubation, the floating cells were collected and the attached cells were collected by trypsinization of the cell monolayer. The trypsinized cells were pooled with the floating cells. The cells were washed with PBS twice and fixed in $70 \%$ ethanol for at least $1 \mathrm{~h}$ at $4^{\circ} \mathrm{C}$. The cells were then stained with DNA-binding buffer. The fluorescence profiles of the stained cells were analyzed using the FACSCalibur Flow Cytometer (BectonDickinson). The laser source of a wavelength $488 \mathrm{~nm}$ was used for excitation, and the fluorescence signal was detected using the FL-2 channel. At least 10000 events were counted. The proportion of DNA was analyzed using the Cell Quest and the Modfit LT Version 3.0 Software.

MTT assay. The MTT assay was conducted using a MTT Assay kit from Chemicon International (Temecula, CA) according to the protocol provided by the manufacturer. In brief, $2 \times 10^{3}$ cell clones with stable shRNA transfection were plated with RPMI-1640. Untransfected MCF-7 cells were used as control. After culturing for 10 days, $10 \mu 1$ of MTT $(5 \mathrm{mg} / \mathrm{ml}$ 
in PBS) were added to each well and incubated at $37^{\circ} \mathrm{C}$ for $4 \mathrm{~h}$. After dissolving the resulting formazan product with acid isopropanol, the absorbance was read on a BT 2000 Microkinetics Reader (Bio-Tek Instruments, Inc.) at a wavelength of $490 \mathrm{~nm}$.

Transwell assay. Cells were plated in 24-well transwell units with polycarbonate filters (Coster Corp.) containing $8 \mu \mathrm{m}$ pores. Cells were plated at a density of $1.0 \times 10^{5}$ cells/well in $0.5 \mathrm{ml}$ of serum-free medium. The outer chambers were filled with the increasing concentrations of CCL5 from 0.01 to $100 \mathrm{ng} / \mathrm{ml}$. After $24 \mathrm{~h}$ incubation at $37^{\circ} \mathrm{C}$, the cells were fixed in methanol and stained with haematoxylin. Cells above the filter were gently removed with a cotton swab, and the cells on the underside of the filter were counted on glass slides, and images corresponding to the entire membrane surface were captured using an Olympus inverted microscope equipped with a CCD camera. Values were normalized with the control group in each experiment.

Rhodamine phalloidin. Cells were plated overnight on cover slips, fixed in $3.7 \%$ formaldehyde/phosphate-buffered saline, permeabilized with $0.1 \%$ Triton X-100 and blocked for 30 min with $1 \%$ bovine serum albumin. Cells were incubated with Rhodamine phalloidin for $20 \mathrm{~min}$. Preparations were observed using a Leica TCS SP2 confocal microscope (Leica Microsystems, Richmond Hill, Ontario, Canada) in the basic medical institute of the Medical College of Wuhan University. Image acquisitions were processed using Adobe Photoshop software.

Data analysis. All experiments were repeated at least three times. The data are expressed as mean \pm SEM and were analyzed using one-way analysis of variance (ANOVA) and the Student-Newman-Keul's test for individual comparisons. Student's t test was used to evaluate the differences between the experimental and control groups. A probability value of $\mathrm{P}<0.05$ was considered statistically significant.

\section{Results}

Specific inhibition of ezrin expression by ezrin shRNAs. The ezrin-targeting shRNAs were designed based on a previous work to define the shRNA target sequences, which were chosen from the 553-571 nt and 995-1013, 19 nt base pairs of the coded region of ezrin mRNA. As a control, an empty plasmid pSUPER was used. The ezrin shRNA recombinants were identified by enzyme digestion and sequencing (data not shown). After MCF-7 cells were transfected with ezrin shRNAs recombinants, MCF-7 clones stably expressing ezrin shRNAs were selected and further ezrin expression was identified by RT-PCR and Western blot analyses. As showed in Fig. 1B, Western blot analysis of whole cell lysates revealed a reduction of $70 \%$ in ezrin protein levels, and a $>85 \%$ in ezrin mRNA levels in Fig. 1A without any significant modulation in the amount of GAPDH or B-actin used as the control comparing with the control cells stably expressing pSUPER. As showed in Fig. 1B, the expression of ezrin in MCF-7 clones with stable ezrin shRNA (995) was reduced significantly compared with the MCF-7 clones with stable ezrin shRNA (553).
A

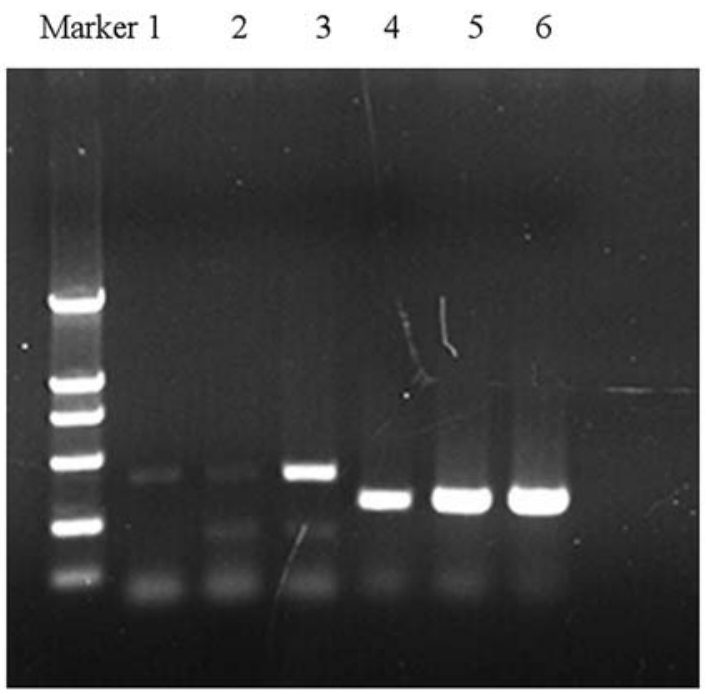

B

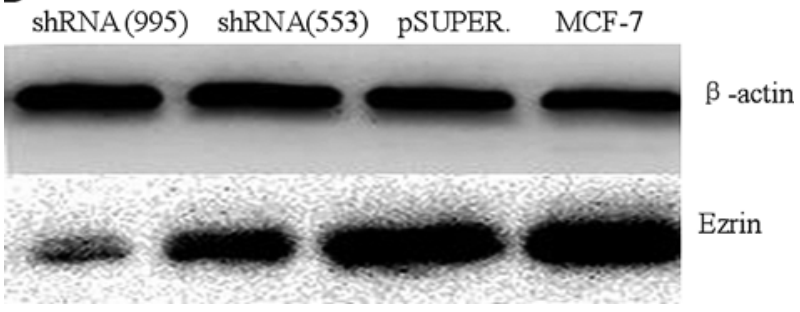

Figure 1. Specific silencing of Ezrin mediated by stably expressed ezrin shRNA. (A) RT-PCR analysis of total RNA of MCF-7 cells stably expressing ezrin shRNA or empty vector pSUPER. The figure shows a representative result of three independent experiments. 1-3, ezrin expression in shRNA (553), shRNA (995) and pSUPER; 4-6, GAPDH expression. (B) Western blot analysis of whole-cell lysates of MCF-7 cells stably expressing ezrin shRNA or empty vector pSUPER. Cells were analysed by immunoblotting with specific antibodies to ezrin and $\beta$-actin.

Silencing ezrin expression by shRNA inhibits the proliferation of MCF-7 cells. We observed the biological behavior of MCF-7 cells when the expression level of ezrin was lowered by shRNA (995). MTT method was used to detect the proliferation of the cells. As shown in Fig. 2, downregulation of ezrin expression distinctly decreased the proliferation rates of MCF-7 cells. The apoptotic index increased dramatically in the shRNA transfected groups compared with the control group on the day $5(\mathrm{t}=4.479, \mathrm{P}<0.01)$. To elucidate the mechanism by which down-regulation of ezrin expression leads to the inhibition of cellular proliferation in MCF-7 cells, fluorescence activated cell sorting (FACS) was performed. Analysis of cell cycle distribution revealed that ezrin downregulation by shRNA primarily affects cell transition from G1 to S-phase in MCF-7 cells. After RNAi treatment, the cell proportion in $\mathrm{G} 2$ phase decreased significantly from $37.6 \pm 5.3 \%$ to $13.3 \pm 4.6 \%(\mathrm{t}=-5.997, \mathrm{P}<0.01)$ (Table I).

Silencing of ezrin reverses the CCL5-induced migration and invasiveness in MCF-7 cells. Increased cell motility and invasiveness of carcinoma cells are key steps in the metastatic cascade. We examined the role of ezrin in the CCL5-induced cell motility and invasiveness in MCF-7 cells. Transwell 


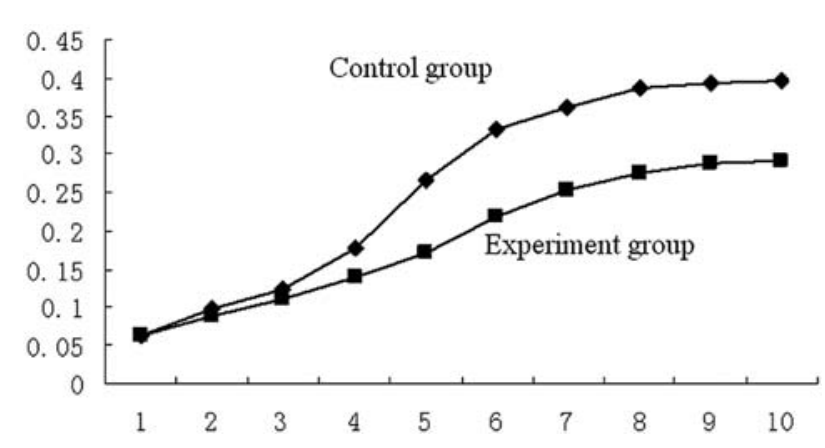

Figure 2. Silencing ezrin expression by shRNA inhibits the proliferation of MCF-7 cells. MTT analysis of MCF-7 cells stably expressing ezrin shRNA (995) or empty vector pSUPER for ten days. The figure shows a representative result of three independent experiments.

Table I. Analysis of cell cycle of MCF-7 cells stably expressing ezrin shRNA(995) or empty vector pSUPER.

\begin{tabular}{lcc}
\hline Cells & G1 $(\%)$ & G2 $(\%)$ \\
\hline Empty vector pSUPER & $39.4 \pm 2.5$ & $37.6 \pm 5.3$ \\
shRNA (995) & $53.9 \pm 1.6$ & $13.3 \pm 4.6$ \\
$\mathrm{t}$ & 7.924 & -5.997 \\
P-value & $<0.01$ & $<0.01$ \\
\hline
\end{tabular}

Decreasing ezrin expression by ezrin shRNA in MCF-7 cells affects cell transition from G1 to S-phase in MCF-7 cells. After RNAi treatment, the cell proportion in G2 phase decreased significantly from $37.6 \pm 5.3 \%$ to $13.3 \pm 4.6 \%(\mathrm{t}=-5.997, \mathrm{P}<0.01)$.

assays were carried out using matrigel-coated transwell culture chambers to evaluate the cell motility in MCF-7 clones stably expressing ezrin shRNAs. After $24 \mathrm{~h}$, invading cells were counted using image analysis. The results shown in Fig. 3 demonstrate that CCL5 induces MCF-7 migration in a dose- and gradient-dependent manner. The optimal concentration of chemokine CCL5 required for migration varied with the expression of ezrin. CCL5 induced significant migration in MCF-7 cells in 5 out of 5 experiments, with a mean MI of 9.7, with maximal migration observed at a concentration of $20 \mathrm{ng} / \mathrm{ml}$ (Fig. 3). In contrast, stable ezrin shRNA-expressing (995) MCF-7 clones showed the reduced cell invasiveness by 2 -fold compared with the cells transfected with pSUPER $(\mathrm{t}=5.268, \mathrm{P}<0.01)$, with maximal migration observed at a concentration of $50 \mathrm{ng} / \mathrm{ml}$, which is higher than the cells with no transfectant.

The CCL5-induced changes in ezrin protein expression in MCF-7 cells. The results shown in Fig. 3 raise the possibility that ezrin is a downstream effector of CCL5 in the context of invasiveness in MCF-7 cells. To investigate this notion, we examined the expression of ezrin as the active phosphorylated level as well as the total level. After treatment with CCL5 for a longer time, the expression of the phosphorylated ezrin was

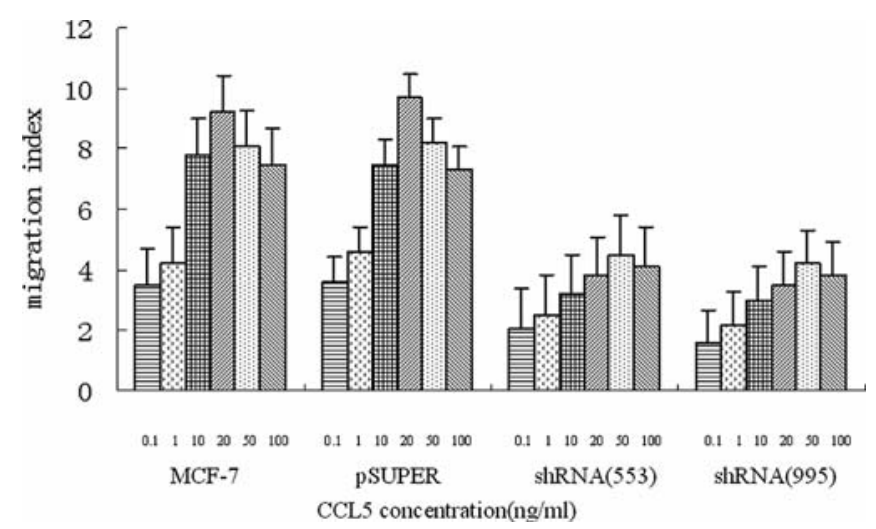

Figure 3. Silencing of ezrin reverses the CCL5-induced metastatic behavior. CCL5-induced invasive capability in MCF-7 cells with stable ezrin shRNA or pSUPER or no transfectant was detected by transwell assay. Migration assays to assess the ability of the CCL5 to induce MCF-7 cells to migrate were performed using a Boyden chamber assay. The CCL5 was added to the lower chamber at increasing concentrations from 0.01 to $100 \mathrm{ng} / \mathrm{ml}$ and cells were added to the upper wells of a 24 -well microchemotaxis chamber, and the migration across a fibronectin-coated polycarbonate membrane $(8 \mu \mathrm{m}$ pore size) was assessed after $24 \mathrm{~h}$. The migrated cells in 5 high power microscopic fields were counted in triplicate wells. Data are expressed as the mean migration index $[\mathrm{MI}=($ Number of cells migrating to test $) /($ Number of cells migrating to negative control)].

markedly increased ( $\mathrm{F}=30.97, \mathrm{P}<0.01)$ (Fig. 4A). Reducing ezrin expression markedly by shRNA inhibited the CCL5induced increasing changes in the total and the phosphorylated ezrin in MCF-7 cells ( $\mathrm{P}>0.05)$ (Fig. 4B). The above results showed that ezrin regulates the CCL5-induced migratory signalling pathway in MCF-7 cells.

Silencing of ezrin inhibits the CCL5-induced actin cytoskeleton organization in MCF-7 cells. Cell migration is strictly regulated by the reorganization of the actin cytoskeleton and focal adhesions. The effect of CCL5 on the F-actin content of MCF-7 cells was investigated using confocal microscopy which allowed us to quantitate rhodamine-labelled phalloidin staining following treatment with CCL5. Studies using confocal microscopy indicate that there is also a redistribution of the F-actin within the cell, after treatment with CCL5 for 60 min (Fig. 5A). Following treatment, intense F-actin staining was visible near the periphery of the cells and in most cases was polarised toward one side of the cell. Therefore, invasive cells tend to be more elongated than non-invasive cells. To study how the changes in ezrin expression influence the reorganization of cytoskeleton, stable ezrin shRNA (995)expressing MCF-7 clones were labeled for F-actin using rhodamine-phalloidin. The investigation of cell shapes showed pointed pseudopodia or polygonal cell shapes with pointed edges, in which brightly stained, longitudinal actin bundles were formed (Fig. 5B). The stable ezrin shRNA-expressing clones had shrunken cell shapes without visible stress fibers.

\section{Discussion}

Recent studies $(30,31)$ emphasized the idea that tumor cell migration and organ-specific metastasis are critically regulated by chemokines and their receptors. There is increasing mass 


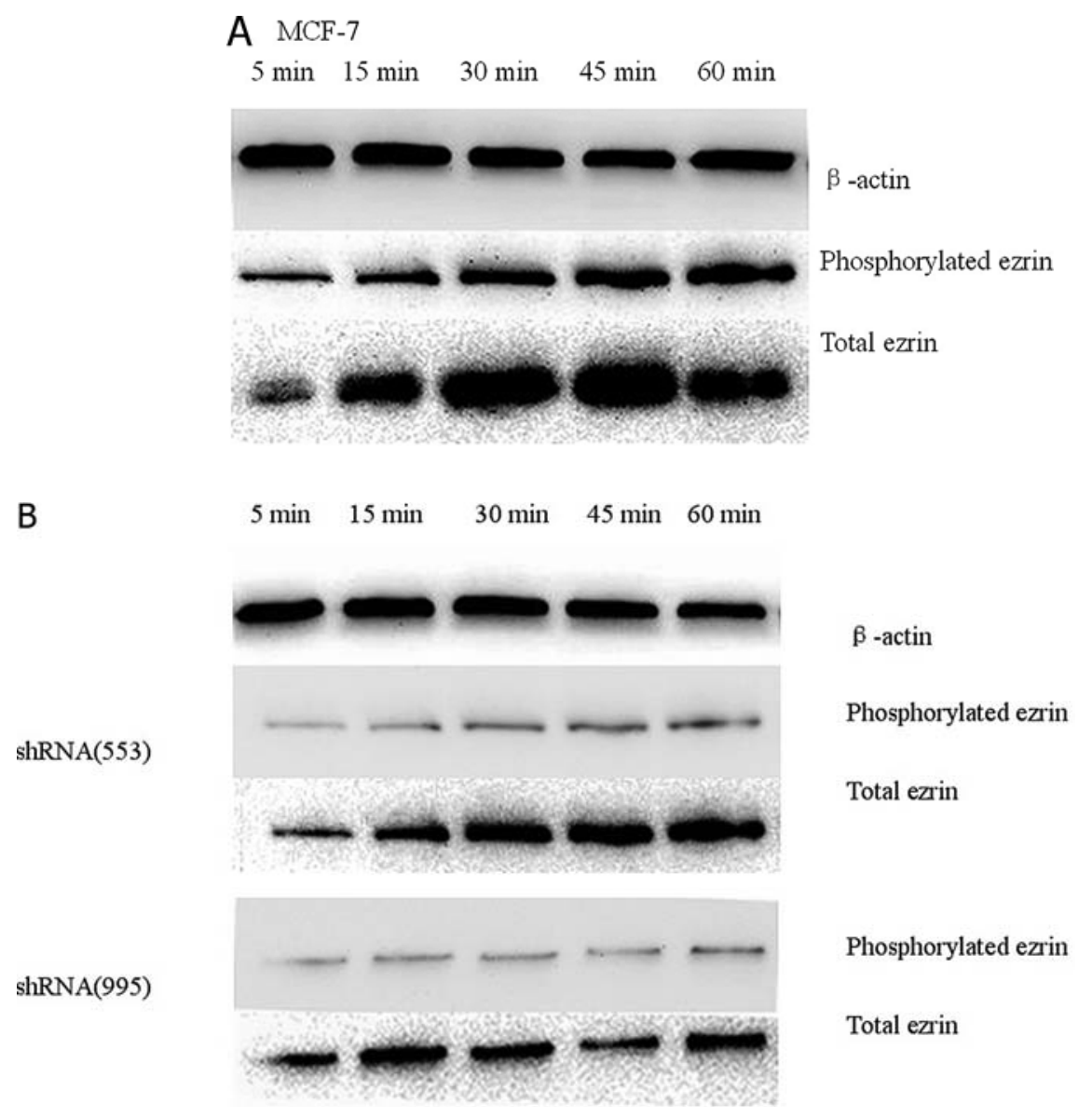

Figure 4. The CCL5-induced changes in ezrin protein expression in MCF-7 cells by Western blotting. (A) CCL5-induced phosphorylated and total ezrin protein expression levels in MCF-7 cell lines were increasing significantly in a time-dependent manner $(\mathrm{F}=30.97, \mathrm{P}<0.01)$. (B) CCL5-induced ezrin protein expression levels in MCF-7 cell lines were increasing only slightly in a time-dependent manner $(\mathrm{P}>0.05)$. The silencing of ezrin by shRNA markedly inhibited the CCL5-induced increacing changes in the total and the phosphorylated levels of ezrin in MCF-7 cells. As shown in (B), the expression of ezrin in MCF-7 clones with stable ezrin shRNA (995) was reduced significantly compared with the MCF-7 clones with stable ezrin shRNA (553).

A

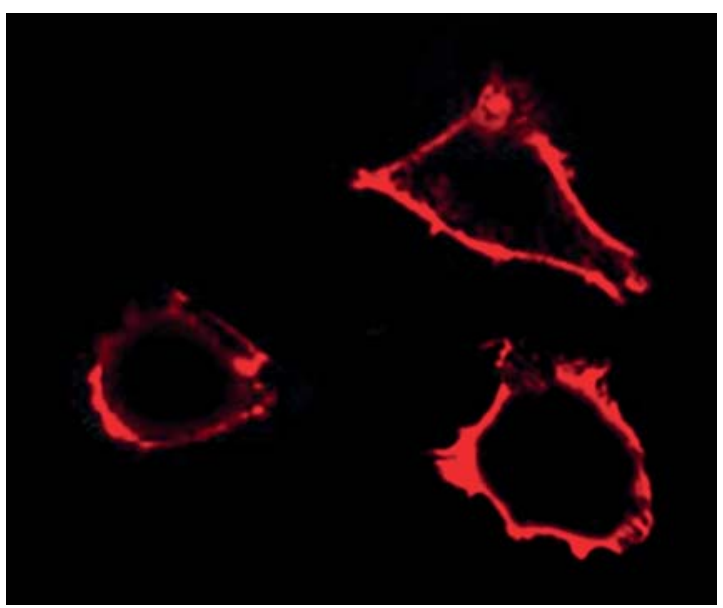

B

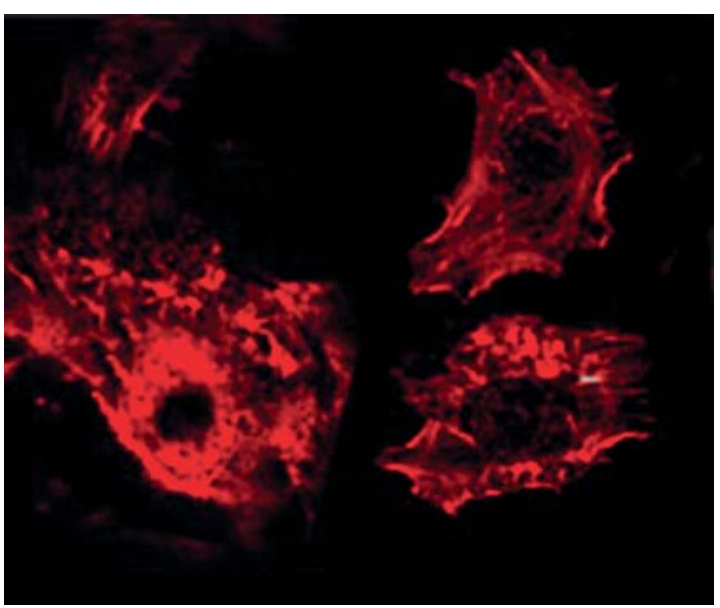

Figure 5. Silencing of ezrin inhibits the CCL5-induced actin cytoskeleton organization in MCF-7 cells. The effect of ezrin silencing on cytoskeleton rearrangement. Representative change in CCL5 (20 ng/ml)-induced cytoskeleton rearrangement (phalloidin-fluorescein isothiocyanate staining) in MCF-7 cells stably expressing ezrin shRNA (A) or pSUPER (B).

of evidence to suggest that chemokine CCL5 and its receptors play a role in the development and progression of several cancer types $(32,33)$. As for breast cancer, previous studies have showed that CCL5 is important in the progression of breast cancer and a correlation between high CCL5 expression and poor prognosis $(5,34,35)$, but its precise function and 
mechanism have not been clarified. A recent study has revealed that antagonists of CCR5, CCR1 or receptors of CCL5, inhibited experimental breast tumor growth (14).

In this study, in order to identify the effects of CCL5 on MCF-7 cell invasive ability, transwell assays were carried out using matrigel-coated transwell culture chambers. To our surprise, the results demonstrate that CCL5 induces MCF-7 invasiveness in a concentration- and time-dependent manner. CCL5 induced significant invasiveness in MCF-7 cells, with a mean MI of 9.7, with maximal invasiveness observed at a concentration of $20 \mathrm{ng} / \mathrm{ml}$. Our findings suggest that CCL5 may play a prominent role in $\mathrm{MCF}-7$ cell migration and invasiveness which could result in the enhanced metastatic propensity. On the other hand, the silencing of ezrin by stably expressed shRNAs reversed the CCL5-induced metastatic behavior of MCF-7 cells, including the expression of the phosphorylation ezrin, the changes in cytoskeleton-mediated cell morphology and the cell motility and invasiveness. In this study, stable ezrin shRNA-expressing MCF-7 clones showed reduced invasiveness by 2 -fold compared with the cells transfected with pSUPER or the cells with no transfectant, with maximal migration observed at a concentration of $50 \mathrm{ng} / \mathrm{ml}$, more than the concentration $(20 \mathrm{ng} / \mathrm{ml})$ for the cells transfected with pSUPER or the cells with no transfectant. Meanwhile, the inhibition of ezrin expression by ezrin shRNA in MCF-7 cells prevented CCL5-induced migration and the phosphorylated ezrin was decreased in these cells.

It is well documented that the loss or inhibition of the function of ezrin, or its associated proteins, results in a reduced gain of the invasive potential. Our results concerning the influence of CCL5 on the expression of ezrin in MCF-7 cells revealed that CCL5 could stimulate the expression and activation of the membrane-cytoskeleton linker ezrin in MCF-7. CCL5 induced a significant increase in the expression of the phosphorylated and the total ezrin protein in MCF-7 cell lines in a time-dependent manner. In fact, ezrin, a key component in tumor metastasis, is mainly required for cell motility, cortical organization and cell division, and the phosphorylated ezrin is the activated form. In the present study, with the maximal invasive ability at $20 \mathrm{ng} / \mathrm{ml}$ of CCL5, ezrin is stimulated to regulate the signal transduction. However, the increasing expression of ezrin induced by CCL5 could be markedly inhibited by shRNA. Stable ezrin shRNA-expressing MCF-7 clones showed reduced cell invasiveness 2-fold compared with the cells transfected with pSUPER or the cells with no transfectant, with maximal migration observed at a concentration of $50 \mathrm{ng} / \mathrm{ml}$, which is higher than that of the MCF-7 cells. The results indicate that the expression of ezrin in breast cancer cells has a positive correlation with the invasive ability of MCF-7 cells.

Tumor cell motility is an important step in the intricate process leading to the formation of metastases and tumor cells demonstrating an increased metastastic potential are more motile than non-metastatic tumor cells. The studies have shown that the structure of the actin network relates to the malignant potential of cell lines and determines their level of motility. The motility and F-actin content of non-invasive cells have been compared with invasive cells, in which a high level of actin polymerisation is a prerequisite for pseudopod formation and necessary for the infiltration of these cells into the tissues. Our results using confocal microscopy indicate that a redistribution of the cytoskeletal F-actin occurs in MCF-7 cells after treatment with CCL5 (20 ng/ml) within $60 \mathrm{~min}$, with movement of F-actin toward the periphery of the cell in a polarised manner. These results indicate that there is a rapid polymerization of F-actin following stimulation with CCL5 and a redistribution of the cytoskeletal actin within cells. Our results show that the degradation of ezrin leads to a disruption of the actin cytoskeleton, showing that ezrin, in response to CCL5, plays an important role in pseudopodia extension and is involved in tumor cell scattering and motility through disrupting the actin cytoskeleton in MCF-7 cells.

Our results indicate that the CCL5 can induce migration and cytoskeletal changes in MCF-7 cells in vitro and suggest a potential role for ezrin in the CCL5-induced processes of breast cancer cell migration, invasiveness and possibly metastasis. We demonstrate that ezrin shRNA specifically inhibits ezrin expression in MCF-7 cells and deregulates several functions such as cell motility and invasiveness, indicating that ezrin plays a key role in the development of human breast cancer cells. Furthermore, blocking of ezrin function induced an increased apoptosis and a proliferation inhibition. It is suggested that ezrin may act as downstream effector of CCL5 and a new anti-metastatic therapeutic target for human breast cancer.

Nevertheless, animal experiments should be conducted to verify the functions of CCL5 in inducing invasiveness and mechanism of interaction between CCL5 and ezrin as well as the consequent signaling pathway should also be clarified.

\section{Acknowledgements}

This study was supported by the Natural Science Foundation of Hubei Province No.301130851, the 11th Five Years Key Programs for Science and Technology Development of China Hubei Province No.S05-1 and the Research Foundation of Public Health Bureau of Hubei Province No.JX4B19.

\section{References}

1. Nicolson GL: Paracrine and autocrine growth mechanism in tumor metastasis to specific sites with particular emphasis on brain and lung metastasis. Cancer Metastasis Rev 12: 325-343, 1993.

2. Lévesque J-P, Hendy J, Takamatsu Y, et al: Disruption of the CXCR4/CXCL12 chemotactic interaction during hematopoietic stem cell mobilization induced by GCSF or cyclophosphamide. Clin Invest 111: 187-196, 2003.

3. Youngs SJ, Ali SA, Taub DD and Rees RC: Chemokines induce migrational responses in human breast carcinoma cell lines. Int J Cancer 71: 257-266, 1997.

4. Muèller A, Homey B, Soto H, et al: Involve-ment of chemokine receptors in breast cancer metastasis. Nature 410: 50-56, 2001.

5. John AE, Berlin AA and Lukacs NW: Respiratory syncytial virus-induced CCL5/RANTES contributes to exacerbation of allergic airway inflammation. Eur J Immunol 33: 1677-1685, 2003.

6. Azenshtein E, Luboshits G, Shina S, et al: The CC chemokine RANTES in breast carcinoma progression: regulation of expression and potential mechanisms of promalignant activity. Cancer Res 62: 1093-1102, 2002.

7. Stormes KA, Lemken CA, Lepre JV, et al: Inhibition of metastasis by inhibition of tumor derived CCL5. Breast Cancer Res Treat 89: 209, 2005. 
8. Zhang Y, Yao F, Yao X, Yi C, Tan C, Wei L and Sun S: Role of CCL5 in invasion, proliferation and proportion of CD44 ${ }^{+} / \mathrm{CD} 24$ phenotype of MCF-7 cells and correlation of CCL5 and CCR5 expression with breast cancer progression. Oncol Rep 21: 1113-1121, 2009.

9. Wigler N, Shina S, Kaplan O, et al: Breast carcinoma: a report on the potential usage of the CC chemokine RANTES as a marker for a progressive disease. IMAJ 4: 940-943, 2002.

10. Yaal-Hahoshen N, Shina S, Leider-Trejo L, et al: The chemokine CCL5 as a potential prognostic factor predicting disease progression in stage II breast cancer patients. Clin Cancer Res 12: 4474, 2006.

11. Soria G and Baruch AB: The inflammatory chemokines CCL2 and CCL5 in breast cancer. Cancer Lett 267: 271-285, 2008.

12. Luboshits G, Shina S, Kaplan O, Engelberg S, Nass D, LifshitzMercer B, Chaitchik S, Keydar I and Ben Baruch A: Elevated expression of the $\mathrm{CC}$ chemokine regulated on activation, normal $\mathrm{T}$ cell expressed and secreted (RANTES) in advanced breast carcinoma. Cancer Res 59: 4681-4687, 1999.

13. Karnoub AE, Dash AB, Vo AP, et al: Mesenchymal stem cells within tumour stroma promote breast cancer metastasis. Nature 449: 557-563, 2007.

14. Robinson SC, Scott KA, Wilson JL, et al: A chemokine receptor antagonist inhibits experimental breast tumor growth. Cancer Res 63: 8360-8365, 2003.

15. Sato N, Funayama N, Nagafuchi A, Yonemura S and Tsukita S: A gene family consisting of ezrin, radixin and moesin. Its specific localization at actin filament/plasma membrane association sites. J Cell Sci 103: 131-143, 1992.

16. Shaw RJ, Henry M, Solomon F and Jacks T: RhoA-dependent phosphorylation and relocalization of ERM proteins into apical membrane/actin protrusions in fibroblasts. Mol Biol Cell 9: 403-419, 1998 .

17. Takahashi K, Sasaki T, Mammoto A, Hotta I, Takaishi K, Imamura H, Nakano K, Kodama A and Takai Y: Interaction of radixin with Rho small $\mathrm{G}$ protein GDP/GTP exchange protein Dbl. Oncogene 16: 3279-3284, 1998.

18. Gautreau A, Poullet P, Louvard D and Arpin M: Ezrin, a plasma membrane-microfilament linker, signals cell survival through the phosphatidylinositol 3-kinase/Akt pathway. Proc Natl Acad Sci USA 96: 7300-7305, 1999.

19. Algrain M, Turunen O, Vaheri A, Louvard D and Arpin M: Ezrin contains cytoskeleton and membrane binding domains accounting for its proposed role as a membrane-cytoskeletal linker. J Cell Biol 120: 129-139, 1993.

20. Vaheri A, Carpen O, Heiska L, Helander TS, Jaaskelainen J, Majander-Nordenswan P, Sainio M, Timonen T and Turunen O: The ezrin protein family: membrane-cytoskeleton interactions and disease associations. Curr Opin Cell Biol 9: 659-666, 1997.

21. Curto M and McClatchey AI: Ezrin: a metastatic determinant? Cancer Cell 5: 113-114, 2004.

22. Hunter KW: Ezrin, a key component in tumor metastasis. Trends Mol Med 10: 201-204, 2004.
23. Yu Y, Khan J, Khanna C, Helman L, Meltzer PS and Merlino G: Expression profiling identifies the cytoskeletal organizer ezrin and the developmental homeoprotein Six-1 as key metastatic regulators. Nat Med 10: 175-181, 2004.

24. Khanna C, Wan X, Bose S, Cassaday R, Olomu O, Mendoza A, Yeung C, Gorlick R, Hewitt SM and Helman LJ: The membranecytoskeleton linker ezrin is necessary for osteosarcoma metastasis. Nat Med 10: 182-186, 2004.

25. Makitie T, Carpen O, Vaheri A and Kivela T: Ezrin as a prognostic indicator and its relationship to tumor characteristics in uveal malignant melanoma. Invest Ophthalmol Vis Sci 42: 2442-2449, 2001.

26. Tokunou M, Niki T, Saitoh Y, Imamura H, Sakamoto M and Hirohashi S: Altered expression of the ERM proteins in lung adenocarcinoma. Lab Invest 80: 1643-1650, 2000.

27. Elliott BE, Meens JA, SenGupta SK, Louvard D and Arpin M: The membrane cytoskeletal crosslinker ezrin is required for metastasis of breast carcinoma cells. Breast Cancer Res 7: R365-R373, 2005.

28. Li Q, Wu M, Wang H, Xu G, Zhu T, Zhang Y, Liu P, Song A, Gang C, Han Z, Zhou J, Meng L, Lu Y, Wang S and Ma D: Ezrin silencing by small hairpin RNA reverses metastatic behaviors of human breast cancer cells. Cancer Lett 261: 55-63, 2008.

29. Ohtani K, Sakamoto H, Rutherford T, Chen Z, Satoh K and Naftolin F: Ezrin, a membrane-cytoskeletal linking protein, is involved in the process of invasion of endometrial cancer cells. Cancer Lett 147: 31-38, 1999.

30. Al-Hajj M, Wicha MS, Benito-Hernandez A, et al: Prospective identification of tumorigenic breast cancer cells. Proc Natl Acad Sci USA 100: 3983-3988, 2003.

31. Kondo T, Setoguchi T and Taga T: Persistence of a small subpopulation of cancer stem-like cells in the C6 glioma cell line. Proc Natl Acad Sci USA 101: 781-786, 2004.

32. Mrowietz U, Schwenk U, Maune S, Bartels J, Kupper M, Fichtner I, Schroder JM and Schadendorf D: The chemokine RANTES is secreted by human melanoma cells and is associated with enhanced tumuor formation in nude mice. Br J Cancer 79: 1025-1031, 1999.

33. Fischer M, Juremalm M, Olsson N, Backlin C, Sundstrom C, Nilsson K, Enblad G and Nilsson G: Expression of CCL5/ RANTES by Hodgkin and Reed-Sternberg cells and its possible role in the recruitment of mast cells into lymphomatous tissue. Int J Cancer 107: 197-201, 2003.

34. Manes S, Mira E, Colomer R, Montero S, Real LM, GomezMouton C, Jimenez-Baranda S, Garzon A, Lacalle RA, Harshman K, Ruiz A and Martinez A: CCR5 expression influences the progression of human breast cancer in a p53dependent manner. J Exp Med 198: 1381-1389, 2003.

35. Niwa Y, Akamatsu H, Niwa H, Sumi H, Ozaki Y and Abe A: Correlation of tissue and plasma RANTES levels with disease course in patients with breast or cervical cancer. Clin Cancer Res 7: 285-289, 2001. 